\title{
UNA APROXIMACIÓN AL CONCEPTO DE GERENCIA Y ADMINISTRACIÓN APLICADO A LA DISCIPLINA DE ENFERMERÍA
}

\author{
Uma aproximação ao conceito de gerência e administração aplicado à disciplina de \\ enfermagem \\ An approach to the concept of management and administration applied to the \\ discipline of nursing
}

Joana Hernández Ortiz ${ }^{1}$

Danelia Gomez Torres ${ }^{2}$

\begin{abstract}
RESUMEN
El presente trabajo fue desarrollado mediante la indagación de términos en revistas virtuales en medios electrónicos. El objetivo fue obtener información de las definiciones de gerencia y administración; Al mismo tiempo, realizar la interpretación de datos y efectuar una crítica de cada una de las definiciones seleccionadas, contrastando con los conocimientos de enfermería, ya que estos están forjados dentro del área empresarial. Se utilizó una metodología basada en constructos; aplicados para reeducar deficiencias terminológicas. Al analizar cada una de estas definiciones, se identificó que la gerencia y la administración coinciden en algunos aspectos y al aplicarlos logran confundir, aún teniendo similitudes en la práxis, no realizan las mismas acciones, ya que la gerencia es la estrategia y la administración es la ejecución. De esta manera, los resultados obtenidos fueron dos conceptos aplicables a la disciplina de Enfermería. Concluyendo así, que una disciplina necesita el apoyo de otras áreas del conocimiento.
\end{abstract}

Palabras clave: Enfermería. Conocimiento. Gerencia.

\section{Resumo}

0 presente trabalho foi desenvolvido diante da indagação dos términos em periódicos virtuais em médios eletrônicos. 0 objetivo foi obter informação das definições de gerência e administração e, ao mesmo tempo, realizar a interpretação de dados e efetuar uma crítica de cada uma das definições selecionadas, contrastando com os conhecimentos de enfermagem, já que estes geralmente ficam forjados dentro da área empresarial. Utilizou-se a metodologia baseada nos constructos, aplicada para reeducar deficiências terminológicas. Ao analisar cada um destas definições, identificou-se que a gerência e a administração coincidem em alguns aspectos e têm similitudes, mas, na prática, não realizam as mesmas ações já que a gerência é a estratégia e a administração é a execução. Desta maneira, os resultados obtidos foram dois conceitos aplicáveis à disciplina de Enfermagem, concluindo que toda disciplina precisa de outras áreas de conhecimento.

Palavras chave: Enfermagem. Conhecimento. Gerência.

\begin{abstract}
This work was developed by the research of terms or concepts in virtual magazines or electronic media. The objective was to obtain the information of management and administration definitions and, at the same time, perform the interpretation of data and criticizing each of the settings, contrasting them with nursing knowledge, since these are often forged within the business. It was used a methodology which is based in constructs, applied to re-educate terminological deficiencies. By analyzing each of these definitions, it was identified that the management and the administration have similarities in their meanings, when applied can be easily confused, while having similarities in the praxis, they don't perform the same actions as management is the strategy and administration is execution. In this way, the results obtained were two concepts applicable to the nursing discipline, concluding that every discipline requires the support and the knowledge of other areas.
\end{abstract}

Keywords: Nursing. Knowledge. Management

\footnotetext{
'Pasante de la Licenciatura de Enfermería. Realizando Servicio en la Facultad de Enfermería y Obstetricia de la Universidad Autónoma del Estado de México, Integrante y Colaboradora de la Revista Horizontes en Salud Expresión de Enfermería.Toluca- Estado do México-México. E-mail: satelite866@hotmail.com, 2Doctora por la EEAN-UFR, coordinadora de investigación de la FEYO de la UAEM, miembro del cuerpo académico Ejercicio de Enfermería.Toluca- Estado do México- México. E-mail: gomezdanelia@usa.net
} 


\section{INTRODUCCIÓN}

El presente artículo se crea por la necesidad de encontrar diferencias o similitudes entre los términos gerencia y administración, ya que éstos se relacionan generalmente en el área empresarial, este artículo desarrolla la aplicación y conceptualización de éstos en el área de Enfermería. Se han utilizado desde los inicios de las escuelas de Enfermería y hospitales hasta la actualidad, formando parte del área propiamente, sin embargo, la mayor preocupación que existe se da porque los términos aún no se han conceptualizado dentro del área.

Debemos tomar en cuenta que la existencia de teorías, que son tentativas de explicación, siempre han sido necesarias y esenciales para afirmar el pensamiento. ${ }^{1}$ Dado que es un paso inmanente en una investigación como tal, la construcción científica no puede dejarse en un segundo plano, de ahí el interés de indagar en los medios electrónicos como es el internet, razón por la cual se consideró como fuente de información para esta investigación por contener un alto grado de actualización en comparación de las fuentes impresas.

Al indagar, se busca dar una propuesta de concepto aplicado a Enfermería creando constructos. Estos proporcionarán los ajustes conceptuales para emplear la percepción del sujeto-objeto y la comprensión de las respuestas buscadas, además se necesita crear términos, vocablos, palabras, expresiones que signifiquen causas, propósitos de conocer, medios de identificar la naturaleza para distinguirla entre la opinión corriente en el conocimiento fundamentado. La opinión corriente es atribuida al sentido del saber común de todo, es el saber que no es buscado sistemáticamente, así, el fruto del saber se denomina "doxa". Se pretende que con esta indagación se llegue a tener como resultado una experiencia doxa, en la obtención de nuevos constructos que ayuden a la Enfermería a apropiar su terminología y por ello, subsanar la gran preocupación de que esta área no cuente con sus propios conceptos. La gran mayoría de los profesionales de Enfermería adoptan los conceptos empresariales o de otras áreas que no pertenecen a la salud, por ello la intención de conceptuar gerencia y administración y dar un nuevo significado o ajuste conceptual y ser compatibilizado con el discurso de Enfermería.

Los constructos sirven de apoyo a la idea de incrementar nuevos elementos, a la definición del cuerpo de saber en este caso particular de la disciplina de Enfermería. Se puede observar que el término:

Constructo fue forjado para reeducar deficiencias terminológicas y entender qué significa: Ideas y términos, categorías, principios conductores, opiniones influyentes o conceptos esenciales, adoptados en una teoría o área de estudio. ${ }^{2}$
La función del constructo, es mediar la distinción del objeto como fue percibido, además de facilitar la conceptuación de las relaciones de los sujetos involucrados en la investigación, favoreciendo la delimitación del espacio al alcance de la verdad. ${ }^{1}$

En versión de Carvalho, el ajustar los recursos y determinar palabras y conceptos, sirve para dirigir la investigación en la teoría evolutiva. Los conceptos surgen para enlistar aspectos de estudio dando orden y significado a los términos, con esto se pretende lograr que la Enfermería adopte los términos propuestos, como propios, una vez elaborado el constructo.

Por lo referido, la Enfermería tiene la necesidad de construcción científica ${ }^{1}$ en donde no solo las investigaciones se llevan a cabo por demanda o por retos, si no por hacer que de esta profesión obtenga mayor información, en donde cada profesional cree nuevos conocimientos.

\section{METOLOGÍA}

La selección de la temática surge por la intención de apor tar conceptos de gerenciay administración en el ámbito de la disciplina de Enfermería. Cuyo objetivo fue obtener información de estas definiciones para construir conceptos aplicables a la enfermería.

La palabra constructo es una noción teórica derivada del objeto de interés que se desea medir, planteada usualmente en términos de hipótesis. ${ }^{3}$

Se menciona que los constructos fueron forjados para reeducar deficiencias terminológicas, esto debe ser entendido como ideas, términos, categorías, principios conductores, opiniones influyentes o conceptos esenciales adoptados en una teoría o área de estudio. ${ }^{1}$

Metodológicamente podemos decir que para crear un constructo, se parte de la reflexión, auto discusión, como los filósofos refieren la auto-discusión del espíritu consigo mismo. La reflexión como actividad de pensar sobre la naturaleza sirve también como instrumento de búsqueda de la verdad, apoyada en la lógica y en el orden de los conceptos.

Ante lo referido, para conceptualizar y definir, se asume a la capacidad reflexiva del pensamiento, la condición del ajustar y crear términos para poder hablar con firmeza sobre los casos de nuestro interés, de esta manera, la reflexión de las premisas busca respuestas engarzadas de la verdad.

Para llegar a un constructo se deben determinar categorías (conceptos, definiciones), de esta manera se amplía el poder de la razón, en designar y dividir las cosas, así el pensamiento gana poder de clasificar las relaciones del sujeto con las cosas, donde el pensamiento de la personalidad justifica (por la razón) el conocimiento fundamentado, razón de ser del constructo.

Por otro lado, para obtener información de la metodología para desarrollar en esta temática, se llevó a cabo 
la indagación en medios electrónicos especialmente en revistas de Enfermería como son: La Escuela Anna Nery: revista de Enfermería, y las que se encuentran en la Red de Revistas Científicas de América Latina y el Caribe, España y Portugal, Redalyc y también la biblioteca científica electrónica Online Scielo Chile por ser un medio de divulgación amplio. Se seleccionaron artículos que estén relacionados en las dos categorías en estudio: El área de administración, gerencia, así como filosofía.

Se establece como criterios de inclusión para este estudio, artículos de fuentes electrónicas por concebirse informaciones actualizadas, así como los artículos que respondan a los descriptores de gerencia, conceptualizando gerencia, concepto de administración, conceptualizando administración y constructo metodológico.

En la primera búsqueda de 68 artículos identificados relacionados con el tema, se descartaron 61 quedando 7 artículos, por no contar con las definiciones de interés, se llevó a cabo una segunda búsqueda encontrando 75 artículos de los cuales se discriminaron nuevamente 72 , quedando 3 . Así un total de 10 artículos para la elaboración de esta investigación. Posterior a la selección de los artículos en las fuentes mencionadas, fueron analizados las dos categorías que son: administracióny gerencia para conocer el significado de ambos términos, como producto de la reflexión surgida en las lecturas.

Para el análisis e interpretación de los datos, fue oportuno analizar y efectuar una crítica de cada una de las definiciones seleccionadas y contrastar con los conocimientos disciplinados de la Enfermería. Al concluir se hizo mención de las características de cada término y así, formar un constructo resultando casi el concepto propio aplicable a la disciplina de Enfermería.

\section{CONCEPTUALIZANDO EL TÉRMINO DE GERENCIA}

El concepto de gerencia se ha creado según los antecedentes, por una necesidad del subordinado (trabajador), con el objetivo de ser guiado para realizar con éxito sus actividades. Dentro del área de la salud se usa este término aplicado como un sinónimo de administración, al tener coincidencia en los pasos, las definiciones que se presentarán no son aplicables ampliamente en esta área, pero son utilizadas por cultura dentro de este campo, sólo son aplicables en el área empresarial. Sin embargo en el ámbito de la salud, se caracteriza por el uso de otro término denominado atención gerenciada (managed care) que es la forma organizativa que surge como respuesta a la crisis en los sistemas de salud. ${ }^{4}$

En esta definición no se menciona un concepto como tal, sin embargo se hace énfasis de que fue creado a causa de una crisis en los sistemas de salud, por ello la llama atención gerenciada, se concibe como en una forma organizativa, presentándola a continuación:
La gerencia y gestión vienen de la raíz gesto, que procede del latín gestos, definido como actitud o movimiento del cuerpo, el cual a su vez se deriva de genere, que significa llevar, conducir, llevar a cabo ${ }^{5}$

Fragmentando el significado real de la palabra gerencia, se da un ejemplo muy claro de las personas, que para poder moverse 0 poder realizar alguna actividad como tomar un objeto, realizamos un movimiento con el brazo, esto se hace en dirección al artículo con el objetivo de poseer.

Entonces en una institución al aplicar el término gerencia a un determinado grupo de subordinados para poderlos dirigir, primero se debe considerar un logro del objetivo que nos lleve a la meta propuesta y con ello lograr el éxito.

Se puede mencionar que la administraciónes una forma de coordinar los bienes y es más operativo, sin embargo la gerenciatiene un carácter directivo y estratégico como se puede ver en la siguiente definición:

La gerencia es como aquel proceso donde se relaciona un conjunto de elementos que aseguran la óptima utilización de un activo que siempre ha estado presente en la empresa, pero que no había sido debidamente aprovechado. ${ }^{6}$

Este término hace una observación donde menciona que la función de la gerencia no ha sido aprovechada en un $100 \%$, más bien existe desconocimiento de todas las funciones que debe de tener; la gerenciaes una acción social desarrollada por el propio gerente en un ámbito especial y en una temporalidad en la que él se encuentra. ${ }^{7}$ En otras palabras, está encargada de guiar a sus subordinados pero también de respetar los derechos y obligaciones del trabajador, es acertado el hacer mención de que es una acción social porque trabaja con sus subordinados para lograr así una meta y de acuerdo a la normatividad de las instituciones, tiene un determinado tiempo en el puesto para ejercer, llevando a cabo las actividades y además se da una connotación disciplinaria como se menciona a continuación:

Gerencia es como una multidisciplina desprovista de condicionamientos sociales y como una entidad autónoma que soslaya el carácter epistemológico de la administración como ciencia de la dirección. $7: 72$

De acuerdo con este término, al mencionar que la gerencia es multidisciplinaria, nos lleva a identificar que el gerente se debe de encargarse de utilizar estrategias para obtener un mejor desempeño de sus subordinados, en todas los áreas del organismo social, además de que la persona que está a cargo de gerenciar debe tener un conocimiento de la forma correcta en que deben realizar cada una de las 
actividades de sus subordinados y poseer una autoridad ante ellos.

Hay que considerar que la gerencia lleva una serie de pasos para lograr el éxito haciendo uso de recursos físicos, financieros y del factor humano; si no contara con la mano de obra, si no se contara con recursos físicos los subordinados con que trabajarían, si no tuviera los recursos financieros con que obtendrían ingresos para obtener los recursos físicos y pagar a los subordinados. Entonces la gerencia, la institución, el subordinado, el recurso físico y financiero es un complemento o concepción de un equipo de trabajo, que va en busca de un beneficio económico. La gerencia es como un arte, una ciencia que se ocupa del uso correcto, provechoso y sistemático de todos los recursos de una empresa, dicho de otra manera:

La gerencia es el arte y ciencia de trabajar con y a través de un equipo de personas hacia el logro de los objetivos de una organización. Esto implica construir un cuerpo de conocimiento sobre dicha actividad, y que la actividad del gerente involucre relación con otras personas para lograr los objetivos de la organización. ${ }^{8}$

Entonces la gerencia es la base de una institución o empresa porque viene siendo el manejo estratégico de la organización, ya que su función es hacer el uso correcto de sus recursos y además es un arte, porque no todos cumplen con las características para poder ejercer la gerenciay yi no se llegase a usar de manera adecuada, lo que puede suceder es que la institución llegue a la quiebra por un mal uso de sus recursos. Entonces como la gerencia sólo puede ser manejada por una persona al fusionarse ambos términos se dice que:

La gerencia del hombre organizacional es el desempeño adecuado de una organización, se hace posible por el equilibrio de las necesidades de realizar su trabajo y mantener un nivel satisfactorio entre la distribución y el entusiasmo de las personas. $^{9}$

En este caso se menciona porque al no existir esta persona apta o con cualidades organizativas no se podría ejercer con eficiencia. Es muy clara esta versión porque la persona apta para el puesto de gerente debe de equilibrar las necesidades de la empresa, de su subordinado y aprovechar al máximo la producción, porque ambos van en busca de un recurso financiero y para lograr que el subordinado haga bien su trabajo, éste debe de buscar estrategias para elevar el entusiasmo de los trabajadores.

Con lo ya plasmado, se resume que: la gerencia es el elemento clave en el desempeño y supervivencia organizacional. $^{10}$
Además se dice que la gerencia es una acción social y que sólo se da por temporadas de acuerdo con el ámbito social, sin embargo, discordamos con esa posición porque no puede ser una acción temporal ya que la gerencia se da todo el tiempo. El profesionista que está a cargo de gerenciar, no puede utilizar la gerencia a su conveniencia o de acuerdo a sus necesidades, la debe de usar de acuerdo a las necesidades de la institución y de sus trabajadores, al mismo tiempo aprovechar al máximo los beneficios y oportunidades que la institución otorga a sus trabajadores.

\section{CONCEPTUALIZANDO EL TÉRMINO DE ADMINISTRACIÓN}

La administraciónes uno de los términos que más ha sido utilizado en el área empresarial y en las instituciones, sin embargo no existe una definición o concepto de administración en Enfermería propiamente dicho. En este apartado se busca llegar al constructo que se acerque al ámbito en Enfermería, ya que al indagar en los medios electrónicos, nos percatamos que los conceptos de administración sólo están definiendo la palabra administración, siendo que únicamente la define de una manera general. Entonces la administración es la actividad humana; consiste en ejecutar los procesos ya mencionados de planear, organizar, dirigir, coordinar y controlar. 5:118

Ante lo referido, la administración es manejada por el factor humano, como ejemplo podemos mencionar que las computadoras o los robots que en la actualidad se han creado como grandes avances de la ciencia, no pueden pensar, únicamente obedecen órdenes, las personas que están capacitadas pueden ejercer estas actividades que se mencionan como: planear, organizar, dirigir, coordinar y controlar. Considerando el término según el Diccionario Real de la Academia Española, éste define a la administración en varios sentidos, como el político, religioso, médico y organizativo. ${ }^{10: 114}$

En esta fuente de información, el concepto de administración existe, pero no explica con detalle su significado, sólo menciona las áreas donde es utilizado, en realidad no es aplicado solamente en las áreas que menciona, de hecho en todas las entidades de la vida diaria como en el área de trabajo, en la iglesia, en el ejército, en el hogar etc., se menciona que la palabra administración, que viene del latín administrare y ministare, con la acepción de gobernar, regir y disponer de bienes. ${ }^{10: 114}$ Este término lo ha definido con palabras muy cortas y apropiando una idea clara ya que al gobernar, se dispone de bienes y al mismo tiempo repartir y dirigir o regir a un grupo de personas, a las que se ha asignado a su cargo con un fin, sin embargo nos podemos percatar que la administración es un cúmulo de funciones y actividades como se mencionan a continuación: 
Administración es dirigir una institución, ordenar, disponer, organizar, en especial la hacienda o los bienes y desempeñar o ejercer un cargo, oficio o dignidad. $5: 114$

Este término es muy amplio porque se encarga de poner un orden en una institución, de disponer de los bienes, de dirigir a los subordinados e informarles los objetivos y las metas a donde se quiere llegar, en cambio en el siguiente concepto menciona que:

La administración se define como el proceso a través del cual se dirigen los recursos humanos, materiales y financieros para la consecución de ciertos objetivos pero, muy especialmente, para mantener complacido al cliente que es el que permite seguir viviendo y desarrollando a la empresa. ${ }^{5: 17}$

Según se manifiesta, la administración hace uso de recursos humanos, materiales y financieros, que son la base para poder llevar a cabo las actividades administrativas; un ejemplo, guiar o buscar que el trabajador se interese en los objetivos porque es uno de los principales puntos para lograr las metas, si no se indaga que el trabajador o subordinado se interese, no trabajará con un objetivo en mente, por más que se busque el éxito, definitivamente no siempre que exista un grupo de personas va a requerir que los guíe una persona capacitada para realizar las funciones, sin embargo:

La administración es el proceso de trabajar con gente y recursos para alcanzar las metas organizacionales. Los buenos gerentes hacen cosas con eficiencia y con eficacia; cuando el proceso administrativo se ejecuta de forma adecuada, comprende una variedad de actividades, como: planeación, organización, dirección y control. Estas actividades básicas son las funciones tradicionales de la administración. 5:117

Como fue mencionado anteriormente, para poder administrar una institución, su principal elemento es el factor humano, mencionando que siempre que exista una persona capacitada y con aptitudes para administrar una institución, tendrá como resultado una institución exitosa, pero si la enfermera que está a cargo de administrar una institución no está capacitada, no sabe hacer el uso correcto de los recursos materiales y de guiar a los subordinados a su cargo, está claro que la institución puede irse a la quiebra.

Al mencionar que la administración es un proceso que lleva una serie de pasos que debe seguir un orden cronológico, al igual que los demás conceptos, toma al factor humano como el principal elemento con el que se trabaja, básicamente se puede decir que:

La administración consiste en dirigir los recursos materiales y humanos hacia los objetivos comunes de la organización. Este proceso se ha dividido de modo tradicional en diversas actividades de gran alcance. El aspecto más técnico y analítico de la administración abarca la fijación de un objetivo para la organización, la planeación de actividades internas que permitan alcanzar los objetivos y el control de esas actividades de manera que los resultados finales sean los deseados. ${ }^{5: 118}$

El planteamiento anterior describe a la administración como aquel proceso que dirige los recursos materiales y humanos hacia los objetivos, es muy cierto que si no se tiene un objetivo para llegar a una meta, no podrá guiarse de una manera correcta al trabajador, hacer el uso adecuado del recurso material. Entonces se puede hacer mención que la administración se encarga de diversas actividades dentro de una institución como la de planificar, si el administrador no planifica la cantidad del personal que va a llevar a cabo las diversas actividades que se les encomienda, es probable que tenga un exceso de personal y el presupuesto que se le asignó para pagarles no se distribuya correctamente, en cambio si tiene escaso personal la producción, ésta sería mínima por la mala orientación o un exceso de trabajo y su rendimiento físico bajaría. El administrador está encargado de guiar al trabajador para alcanzar las metas que se han propuesto en la institución, para ello tiene que planificar, organizar, dirigir, controlar los recursos financieros y el factor humano, además de que tiene que capacitar a los trabajadores que están a su cargo. Aunado a todo lo anterior, la administración es el conjunto común de procesos que, cuando se efectúa debidamente, favorecen la eficacia y la eficiencia organizacional. 10:118

Dicho lo anterior, se ve a la administración desde un punto de vista del logro de la eficiencia, es muy cier to que el que se encarga de administrar una institución y tiene capacidades para realizar esas funciones, debe de hacer uso de estrategias para alcanzar las metas y objetivos propuestos, viéndolo desde otro punto:

La administración, en cuanto conjunto de procesos distinguibles de quienes los llevan a cabo, también puede concebirse como el trabajo efectuado por personas que tienen el título de gerente. $5: 118$

Esto nos permite la ejecución de actividades que se distinguen de la persona que estáa cargo, si no existiera la persona que fuera capaz de administrar la institución no lograría el éxito, pero la siguiente definición nos describe que: 
La administración implica planear, organizar, dirigir y controlar a las personas que trabajan en una organización a fin de que cumplan las metas de ésta. Estas metas orientan las tareas y actividades que se emprenden. ${ }^{5: 118}$

En este caso resaltan más las características administrativas, dirigidas sólo al personal del que está a cargo del que administra, pero hay que recordar que no únicamente es al que labora en una institución, sino también los recursos materiales y financieros, por ello:

La administración es definida como la actividad humana encargada de organizar y dirigir el trabajo individual y colectivo efectivo en términos de objetivos predeterminados. ${ }^{5: 118}$

Entonces, si se describe como una actividad humana, las funciones administrativas realizadas por una sola persona, la cual trabaja en conjunto con sus subordinados, los cuales van a ser dirigirlos hacia el logro de las metas propuestas, sin embargo, la administración es el proceso de planeación organización, dirección y control del uso de recursos para lograr las metas de desempeño. 10:119 En lo anterior se hace énfasis en los elementos del proceso administrativo y uno de los primeros es la planeación, donde se determinarán las acciones que se deben realizar a futuro. En la etapa de organización se realiza una serie de actividades con una estructura pensada y diseñada; en la etapa de dirección, se dirige y se motiva al empleado para que realice las tareas asignadas y finalmente la etapa de control, es el cumplimiento de lo planeado, el logro del cumplimiento de cada uno de estos aspectos busca llegar a las metas propuestas, sin embargo:

La administración es una práctica usualmente esquematizada como el manejo de los recursos de una organización para el logro de sus objetivos, para lo cual se ejercen los elementos administrativos de planear, coordinar organizar, dirigir, controlar. ${ }^{5: 120}$

De acuerdo con este autor, es acertado que la administración es una práctica pero que está esquematizada y que además va en busca de los objetivos, haciendo uso de los recursos, se mencionan que dentro de las actividades administrativas, está la de coordinar las actividades propias de una institución, además integran las actividades de trabajo que realizarán los subordinados a su cargo, el propósito esencial del administrador es guiarlos, para ser eficientes en sus actividades. También se plantea que la administración es una práctica específica que convierte a una multitud en un grupo eficiente, productivo y orientado hacia metas. ${ }^{5: 120}$

En este concepto, se menciona que el encargado de administrar una institución, debe involucrase en un grupo de trabajadores que estarán a su cargo, para una orientación y de esta manera, convertir al grupo de personas con responsabilidades, capaces de ser eficientes en el área laboral. Si el administrador no tuviera la capacidad de hacer que un grupo de personas, sean productivas y que estén en busca del cumplimiento de metas, la institución no lograría sus propósitos, por lo tanto, la administración es la tarea de conducir el esfuerzo y talento de los demás para el logro de resultados. ${ }^{5: 120}$

Por lo mencionado, se visualiza que la administración tiene muchas aristas y se puede decir que son tareas, las cuales no son tareas fáciles, ya que todo tiene un proceso, entonces el administrador debe tener paciencia y don de mando hacia sus subordinados, para ello tiene que esforzarse y además tener el talento. Si definimos el término de administración en palabras claves, con las cuales se puede entender la función, puesto que:

\section{Administración es el proceso de diseñary mantener un entorno en el que trabajando en grupos, los individuos cumplan eficientemente los objetivos especificos. ${ }^{5: 120}$}

En esta definición menciona que para poder dirigir a un grupo de personas, debe de diseñar un plan de trabajo, y para diseñarlo tiene que ver cuáles son los recursos con los que se cuentan, si en el área de trabajo no cuenta con el suficiente personal, el administrador obtendrá un desequilibrio en la producción laboral y con ello no logrará sus objetivos, este concepto es claro, sin embargo no es lo único que desarrolla el ejecutivo de la administración, el administrar es un mundo de actividades en las cuales se debe de preocupar por cumplirlas para llegar al éxito.

La administración desde tiempos remotos hasta la actualidad, plasma los caminos para dirigir a los subordinados y optimizar los recursos materiales, sin embargo no sólo se administran los recursos materiales e individuos para poderlos concluir y llevarlos al éxito, se debe considerar el recurso financiero, ya que éste es la base y soporte de la institución, sin el recurso económico no podría obtener los recursos materiales necesarios y mucho menos el pago a los empleados, entonces si no están estos tres elementos, el administrador no podría lograr dar cumplimiento de los objetivos institucionales, hay que considerar que es un proceso de trabajo con gente y recursos para alcanzar las metas organizacionales, se hace con el fin de alcanzar las metas, todo lleva un proceso, sin embargo sólo hace mención de que hace uso de personas y recursos, la pregunta es ¿Cuáles recursos?. Se cree que para el término más comprendido debe ser más descriptivo, pero no sólo se debe de tomar en cuenta que, hace uso de recursos sino también de actividades de acuerdo a un proceso y no nada más se trabaja con personas, si no con todo lo que lo rodea, tanto al administrador como a la institución. 
Se considera a la administración, una ciencia compuesta. Aquel que ejerce ese cargo, debe ser competente para guiar un grupo de subordinados y además de hacer el uso de técnicas, hacer el uso de principios los cuales les ayuda a crecer como institución, siendo que no se crea por su estructura, se crea por el tipo de personas que laboran en ese lugar, un administrador debe de esforzarse cada día más para que el subordinado sea guiado de una forma correcta, y además buscar que el subordinado se esfuerce por ser cada día mejor en su trabajo y ser reconocido por los demás, a su vez, debe de buscar competir con los demás integrantes de su grupo.

En su gran mayoría, los conceptos mencionan que la administración, es un conjunto de actividades y tareas a realizar. La cual trabaja con tres factores como son los subordinados, los recursos materiales y financieros, además de buscar alcanzar la meta y cumplir con los objetivos propuestos y para ello debe de planear y hacer uso de estrategias para que el grupo de individuos que coordina logre la eficiencia.

\section{ENFERMERÍA, LA ADMINISTRACIÓN Y GERENCIA}

Buscando dar respuesta e intentar comprender y exponer, lo visible de lo invisible en la realidad objetiva de la enfermería en uno de sus campos de dominio y, con la pretensión de incursionar en conocimientos capaces de apoyar la construcción científica, que aporte instrumentos para criticar la concepción de los términos, que se apeguen a la disciplina de enfermería, se efectúo una contrastación de conocimientos; debido a que la gerencia en la disciplina de Enfermería, sólo es concebida dentro del proceso de trabajo de la enfermera, visualizándola en una primera vertiente al individuo y su organización, considerándola como un modelo racional, en el otro el abordaje como de la práctica social e historicidad sin conceptualización propia.

Podríamos decir que la gerencia en Enfermería, busca dar la mejor atención y que las subordinadas se sientan satisfechas de trabajar, con los recursos que proporciona la institución, para dar un mejor servicio al usuario con bases científicas y sus competencias. Sin embargo los objetivos del trabajo gerencial de Enfermería son: La organización del trabajo y los elementos humanos de enfermería, utilizando un conjunto de instrumentos técnicos propios de la gerencia, es decir: planeación y dimensión del personal de Enfermería, selección del personal, educación continua y/o permanente, así como la supervisión y validación del desempeño entre otros. También utilizan medios como: la fuerza de trabajo, los materiales, equipamiento e instalaciones.

En el trabajo de la enfermera, donde su función principal es coordinar al personal de todas las áreas, de acuerdo a la clasificación del paciente como puede ser: por el tipo de diagnóstico, la gravedad y el requerimiento de la atención, ya sea calculado por indicadores según en las categorías siguientes: cuidados mínimos, parciales, directa, intensivo intermedio, intensivo. Además de hacer el cálculo por jornada laboral en cada uno de los turnos tanto matutino, vespertino y nocturno. El cálculo del personal suplente según incidencias; indiscutiblemente debe aplicar la gerencia como estrategia laboral.

Sin embargo la administración en Enfermería, posee condiciones físicas resistentes como son: la rigidez, la mecanización, la extrema normatividad, las rutinas establecidas, la centralización en puestos clave, el manejo de premios y castigos, la vigilancia como medio de control entre otras, estas son algunas de las características más sobresalientes del sistema administrativo de enfermería.

Entonces, la gerencia al ser multidisciplinaria, debido a que conduce a su subordinado, es una acción social, es organizativa, hace uso de recursos del trabajo físico, financiero, del trabajo humano; es un arte, es una ciencia que busca llegar a la meta, hace uso del manejo estratégico, busca el equilibrio de la institución, surge a consecuencia de las crisis económicas; de ahí que la gerencia, al ser multidisciplinaría posibilita el apropiar una conceptualización para la enfermería, permitiendo fundamentar los conocimientos de enfermería.

\section{CONCLUSIONES}

Al término de este análisis concretizamos que: la disciplina de Enfermería a través del tiempo, se ha apoyado en otras áreas del saber, para acrecentar los conocimientos científicos disciplinarios. Por ello y siguiendo la metodología de constructos, pudimos efectuar un análisis de varios conceptos, para aproximarnos a los términos de gerenciay administración y proponer una conceptualización aplicada a la enfermería.

\section{CONSTRUCTO 1}

Al aproximarnos al concepto de gerencia en Enfermería, considerándola como: Una arte, que busca conducir a sus subordinados de manera estratégica, multidisciplinaria y organizativa para brindar una atención de calidad, logrando que haya un equilibrio entre el paciente, los subordinados y la institución.

Por otro lado, hay que reconocer que una gerencia eficiente, hace que se distinga la institución, ya que se dedica a convertir una multitud en un grupo de gente trabajadora, responsable, comprometida con su trabajo, persigue una meta que está propuesta, además conduce esfuerzos y busca talentos, siempre en pos de la eficiencia y eficacia de sus trabajadores, el administrador que está encargado de un grupo de trabajadores les marca el ritmo a éstos, incrementándoles la exigencias.

\section{CONSTRUCTO 2}

Entonces podríamos acercarnos al concepto de la administración en Enfermería y podemos mencionar que: Es la ejecución de las acciones encargadas de hacer más eficientes y 
comprometidas a un grupo de enfermeras, capaces de desarrollar un trabajo individual o colectivo en beneficio de los pacientes, basándose en el proceso administrativo, aplicando principios y técnicas.

La Enfermera administradora debe usar los principios y técnicas del proceso administrativo para dirigir el trabajo individual y colectivo. Para poder realizar este trabajo, la enfermera encargada de administrar debe de gozar de buena salud mental, vigorosa, experiencia laboral, humanismo y sobre todo capaz de resolver problemas personales, así como los de la propia institución.

También se observó que entre los términos de gerencia y administración, existe una gran diferencia, pues la gerencia trabaja de una manera más estratégica, es como si jugáramos ajedrez; antes de tirar se piensa muy detenidamente una estrategia para darle "jaque al rey", con este juego se puede explicar de una manera más clara como trabaja la gerencia, en cambio la administración también coincide con los mismos pasos que la gerencia pero en este caso la administración se orilla más por la acción, es como el juego del dominó, administra las fichas que te tocan al igual que planeas cómo cerrar el juego para que el compañero de juego no gane. Entonces la administración y la gerencia podrán coincidir en los mismos pasos pero no juegan o planean de la misma manera, ya que la gerencia es la estrategia y la administración es la ejecución.
6.Flores M. Gerencia del conocimiento: Su relación con la generación de capacidades innovativas. Revista de Ciencias Sociales. [on-line] 2005 mayo]; [citado 15 feb 2009]; 002(XI) [aprox. 1 tela] Disponible: http:// redalyc.uaemex.mx/redalyc

7.Romero J. Aproximación a una sociología de la gerencia. Gerencia pública y compromiso social. Gestión y Política Pública, [on-line] 2006]; [citado 15 feb 2009]; 001(XV):71-72 [aprox. 1 tela] Disponible en: http:// redalyc.uaemex.mx/redalyc

8.Chirinos A./ Rincon S. Análisis estratégico de la gerencia de investigación desarrollo de los parques tecnológicos. Rev Venezolana de Gerencia. [on-line] 2006 oct/dic; [citado 23 abr 2009]; 36(11) [aprox. 1 tela] Disponivel em: http://redalyc.uaemex.mx/redalyc

9.Marquez M. C. 0 grid gerencial: uma análise descritiva na área da enfermagem. Esc Anna Nery Rev Enferm 2004 2(8): 275-78

10.Caleb A. Gerencia organizacional y sociedad. Rev Espacio Abierto. [on-line] 2000 jul/sept; [citado 15 feb 2009]; 003(9) [aprox. 2 telas] Disponible en: http://redalyc.uaemex.mx/redalyc

\section{REFERÊNCIAS}

1.Carvalho V. Sobre construtos epistemológicos nas ciências - uma contribução para a enfermagm. Rev. Latino-am Enfermagem [on-line] 2003 jul/ago; [citado 23 abril 2009]; 11(4) [aprox. 8 telas]: Disponivel em: http://www.scielo.br/scielo.

2.Carvalho V. Acerca de las bases teóricas, filosóficas, epistemológicas de la investigación científica: el caso de la enfermería. Rev Latino-am Enfermagen [on-line] 2003 nov/dic; [citado 23 abr 2009]; 11(6) [aprox. 15 telas]. Disponible en: http://www.scielo.br/pdf

3.Consiglio E. Waldo H. Nuevos indicadores clínicos. La calidad de vida relacionada con la salud. Rev Medicina (Buenos Aires). [on-line] 2003; [citado 4 jun 2009]; 2(63) [aprox 3 tela]. Disponible en: http:// uww.medicinabuenosaires.com/revistas

4.Molina J. Fortalecimiento gerencial y liderazgo para la protección social. Salud pública Méx [on-line] 2007; [citado 15 feb 2009]; (49) [aprox. 1 tela] Disponivel em: http://redalyc.uaemex.mx/redalyc

5.Torres S. Una visión contemporánea del concepto de administración: Revisión del contexto colombiano. Cuad. Adm. [on-line] 2006 jul/dic]; [citado 15 feb. 2009]; 032(19):114-120 [aprox. 3 telas] Disponible en: http://redalyc.uaemex.mx/redalyc 